\title{
Acknowledgement to Reviewers of Biology in 2017
}

\author{
Biology Editorial Office \\ MDPI AG, St. Alban-Anlage 66, 4052 Basel, Switzerland
}

Published: 11 January 2018

Peer review is an essential part in the publication process, ensuring that Biology maintains high quality standards for its published papers. In 2017, a total of 50 papers were published in the journal. Thanks to the cooperation of our reviewers, the median time to first decision was 24 days and the median time to publication was 64 days. The editors would like to express their sincere gratitude to the following reviewers for their time and dedication in 2017:

\begin{tabular}{ll} 
Adam, Paul & Leoni, Barbara \\
Ambrose, R.P. Kingsly & Levin, Michael \\
Anifandis, George & Lewis, Jesse S. \\
Archer, Simon & Li, Congjun \\
Arredondo, Luis José Delaye & Li, Zhiheng \\
Balestrieri, Rosario & Ligon, Day B. \\
Becker, Donald F. & Longo, Giuseppe \\
Bilbao, Jose Ramon & Lopez, Dolores E. \\
Bjork, Bryan & Luo, Shuai \\
Blackstone, Neil & Lutz, Stefanie \\
Boczek, Nicole & Maiato, Helder \\
Bohutskyi, Pavlo & Mallo, Moises \\
Borger, Pieter & Martin, Bradley \\
Bozzaro, Salvatore & Mazzulli, Joe \\
Brand-Saberi, Beate & Mc Auley, Mark T. \\
Braun, Edward L. & McGuinness, Dagmara \\
Bricknell, Ian & McKim, Kim S. \\
Brown, Robert J. & McLoughlin, Gráinne \\
Brownstein, Zippora (Zippi) & Mehrshahi, Payam \\
Brunetti-Pierri, Nicola & Menger, Fredric \\
Campos, Francisco & Miller, Robert H. \\
Catacuzzeno, Luigi & Minenkova, Olga \\
Chain, Patrick S.G. & Møller, Anders \\
Chandaka, Giri Kumar & Mulero, Victoriano \\
Chen, Bor-Sen & Müller, Peter \\
Chen, Jinfeng & Muller, Ulrich \\
Cheon, Yong-Pil & Navarro, Pilar \\
Chiao, Ying Ann & Nawrot, Robert \\
Christina, Spry & Nemec, Antonia A. \\
& \\
\hline
\end{tabular}


Cimini, Daniela

Colley, Karen J.

Constantinescu, Stefan

Creixell, Teresa Adell

Curtale, Graziella

De Martino, Andrea

Den Hertog, Jeroen

Dimitrakopoulos, Georgios N.

$\mathrm{Du}$, Min

Durston, Antony

Ellis, Ronald E.

Fabre, Pierre

Fainsod, Abraham

Falconer, Robert

Fernández-Méndez, Mar

Figueras Huerta, Antonio

Fracasso, Alessandra

Fuentes, Augustín

Funk, Richard

Gales, Amandine

Gannon, Victor P.J.

Gazouli, Maria

Gebhardt, Christof

Glazier, Douglas S.

Gong, Shanzhong

Green, Sara Marie Ehrenreich

Gualtieri, Paolo

Hantusch, Brigitte

Harduin-Lepers, Anne

Hejnol, Andreas

Hiroi, Noboru

Ho, Samuel

Huang, Yen-Lin

Igamberdiev, Abir

Ishikawa, Takahiro

Ito, Yuji

Jain, Shalu

Jay Ko, ChemYong

Jin, Fan

Jühling, Frank

Kaneko, Gen

Kanno, Yuichiro

Kaski, Juan Pablo
Neumann Andersen, Mathias

Nishimura, Shin-ichiro

Noble, Denis

Paldi, Andras

Palles, Claire

Pandhal, Jagroop

Panico, Antonio

Peracchi, Alessio

Perego, Carla

Pesole, Graziano

Petro, Thomas M.

Pitto, Letizia

Ponnambalam, Sreenivasan

Pritchard, Hugh W.

Ramos-Suárez, Juan Luis

Re, Lamberto

Reschen, Michael Edward

Rijkers, Ger

Risk, Michael J.

Rizos, Dimitrios

Roach, Dwayne

Roig Molina, Francisco Jose

Roli, Andrea

Roos, Wynand P.

Rutishauser, Urs Stephen

Salinas, Irene

Salmon, Edward D.

Sauerwein, Helga

Schibler, Ueli

Schmidt-Ott, Urs

Seligmann, Hervé

Shumskaya, Maria

Sigurdsen, Trond

Sitja-Bobadilla, Ariadna

Smith, Robert

Spanò, Stefania

Stacewicz-Sapuntzakis, Maria

Staehle, Mary

Stockan, Jenni

Stone, Trevor William

$\mathrm{Su}, \mathrm{TaO}$

Suzuki, Yuichiro J.

Syrén, Per-Olof 
Kavaliers, Martin

Kawakami, Junji

Kawashima, Hiroto

Khisamutdinov, Emil

Kieber-Emmons, Thomas

Kiljunen, Saija

Kim, Jongpil

Kondo, Jiro

Korstad, John

Koslicki, David

Koslicki, David

Krämer, Oliver H.

Kunkle, Brian

Lackie, Peter M.

Lafont, Frank

Lange, Miles

Lei, Lei
Szekely, Gyorgy

Taylor, Andrew

Torinsson Naluai, Åsa

Tosic, Predrag

Traer, Elie

Tsounis, Georgios

Varma, Dileep

Visintainer, Roberto

Wang, Kai

Webb, Grahame

Wenger, Amelia

Wichterle, Hynek

Winuthayanon, Wipawee

Witte, Wolfgang

YUEN, Karen Wing Yee

Zammit, Carla

Ziermann, Janine

(C) 2018 by the authors. Submitted for possible open access publication under the terms and conditions of the Creative Commons Attribution (CC-BY) license (http://creativecommons.org/licenses/by/4.0/) 